\title{
Cambio climático y estacionalidad turística en España: Un análisis del turismo doméstico de costa*
}

\author{
ÁNGEL BUJOSA BESTARD \\ Centre de Recerca Econòmica, UNIVERSIDAD DE LAS ISLAS BALEARES, ESPAÑA. E- \\ mail: angel.bujosa@uib.es
}

\author{
JAUME ROSSELLÓ NADAL \\ Centre de Recerca Econòmica, UNIVERSIDAD DE LAS ISLAS BALEARES, ESPAÑA. E- \\ mail: jrossello@uib.es
}

\section{RESUMEN}

El conocimiento actual de los efectos del cambio climático sobre los flujos turísticos internacionales que recibe España, permite vislumbrar un escenario altamente pesimista caracterizado por una pérdida de atractivo turístico. Sin embargo, más allá de estos resultados, la literatura especializada no ha abordado con suficiente profundidad dos factores de gran importancia en este análisis: el componente estacional de los flujos turísticos y el peso del mercado interior. En este sentido, este trabajo utiliza la temperatura promedio de cada temporada turística (alta, media y baja) para analizar el efecto estacional del cambio climático sobre la probabilidad de visitar un determinado destino turístico incluyendo, en este caso, las provincias costeras de la España peninsular. Los resultados obtenidos de la aplicación de un modelo de elección discreta muestran como las provincias costeras más cálidas se verán perjudicadas por un aumento de las temperaturas mientras que las más frías podrían verse beneficiadas, especialmente en temporada alta.

Palabras clave: Cambio Climático, turismo doméstico, estacionalidad, Familitur, turismo de costa.

\section{Climate Change and Tourism Seasonality in Spain: An Analysis of the Domestic Coastal Tourism}

\begin{abstract}
Current knowledge of climate change consequences on international tourism in Spain suggests a highly pessimistic scenario characterized by a loss of tourist attraction. However, beyond these results, the literature has not considered with sufficient depth two important factors in this analysis: the seasonal component of tourist flows and the weight of the domestic market. By applying a discrete choice model, this paper analyzes the role of the average temperature for the three seasons of the year (high, medium and low) as a factor explaining the probability of visiting a particular destination, in this case, the coastal provinces of mainland Spain. The results show that rising temperatures will negatively affect warmer coastal provinces while cooler provinces could benefit, especially in high season.
\end{abstract}

Keywords: Climate Change, Domestic Tourism, Seasonality, Familitur, Coastal Tourism.

Clasificación JEL: L83, Q54

\footnotetext{
"Este trabajo se ha realizado dentro de los proyectos "Climate Change and Impact Research: the Mediterranean Environment" (CIRCE 036961-2) de la Comisión Europea (VI Programa Marco) y "Efectos del cambio climático sobre la demanda turística. Evidencia empirica y medidas de adaptación” (ECO2010-22143) de la Comisión Interministerial de Ciencia y Tecnología.
}

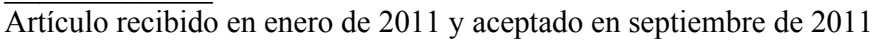

Artículo disponible en versión electrónica en la página www.revista-eea.net, ref. ə-29312 


\section{INTRODUCCIÓN}

El estudio de las consecuencias económicas derivadas del calentamiento global es, sin lugar a dudas, uno de los temas que está suscitando un mayor interés dentro de la literatura especializada de cambio climático. Sectores como la agricultura, con una fuerte dependencia de las condiciones climatológicas y que, al mismo tiempo, gozan de una extensa tradición investigadora en el campo de la economía, han centrado los principales esfuerzos por parte de la literatura especializada. Sin embargo, el turismo, a pesar de su evidente dependencia de las condiciones climáticas, ha quedado relegado a un segundo plano, probablemente por su escasa presencia dentro de la literatura económica.

En cualquier caso, y dejando de lado la literatura estrictamente económica, el vacío en el estudio del turismo como objeto de análisis con relación a los efectos del calentamiento global ha ido cubriéndose en los últimos años. En este sentido, es posible identificar varios estudios dentro de la literatura específica del cambio climático (y marginalmente dentro de algunas publicaciones de turismo) que proporcionan las primeras mediciones del impacto que el calentamiento global puede provocar sobre la actividad turística en términos de la variación del número de turistas. En esta línea, destaca el estudio de Hamilton et al. (2005a) sobre la base de los flujos turísticos internacionales a nivel mundial que estima que el calentamiento global provocará, a medio y largo plazo, una reducción del crecimiento de la actividad turística, a pesar del incremento en el volumen mundial de turistas internacionales que se producirá fruto del aumento de la población y de la renta per cápita.

Con respecto al turismo internacional hacia España, Hamilton et al. (2005a) predicen una fuerte reducción de la cuota de mercado española que pasará a ser del $4.84 \%$ relegándonos desde el segundo lugar actual hasta el sexto lugar en términos de llegadas internacionales. De esta manera, bajo un escenario de cambio climático de un incremento de $1^{\circ} \mathrm{C}$, España sería el tercer país con el mayor efecto negativo (detrás de Méjico y Hong Kong) dentro del grupo de los 25 más importantes.

Estudios similares refuerzan las previsiones de pérdida de cuota de mercado para el turismo internacional hacia España como consecuencia del calentamiento global. De esta manera, a nivel mundial y tomando como referencia las condiciones óptimas para la realización de turismo, Amelung et al. (2007) prevén una importante pérdida de cuota de mercado para la mayor parte de los destinos cálidos y templados del mundo que sería compensada, sólo en parte, por el mayor atractivo de las zonas más septentrionales y meridionales del planeta. Por otra parte, teniendo en cuenta la elevada cuota de mercado que suponen para España los turistas británicos (27.4\%) y alemanes (17.5\%), Maddison (2001), Hamilton y Tol (2007) y Rosselló et al. (2010), encuentran 
que las salidas de turistas internacionales desde estos países frenarán su crecimiento ante diferentes escenarios de cambio climático.

En esta misma línea, pero con un horizonte más lejano, Hein (2007) predice un descenso del 20\% anual en la llegada de turistas a España para el 2080. Aunque las limitaciones de este estudio son patentes al incluir, únicamente, algunas regiones y no considerar los efectos del crecimiento de la renta y de la población, los resultados son ilustrativos al considerar, por primera vez, la heterogeneidad climática de las regiones españolas y la distribución estacional de los turistas a lo largo del año. De esta manera, se prevé que el descenso en la llegada de turistas se acentúe especialmente en verano (con las excepciones de la costa Norte y las Islas Baleares), mientras que durante la primavera y el otoño se incremente sin que, en ningún caso, sea suficiente para compensar las pérdidas registradas durante el verano.

En un intento de profundizar en el estudio de los efectos del cambio climático sobre el mercado turístico desde una perspectiva estacional e incrementar así el conocimiento sobre la distribución temporal y geográfica de los impactos del calentamiento global sobre el mercado turístico, este trabajo pretende analizar los determinantes de la elección de destino turístico sobre la base de un modelo de elección discreta. Más concretamente, y a diferencia de la literatura anterior, este trabajo centra la atención en el mercado interior que, a pesar de no haber sido el centro de atención a nivel internacional, representa, según las Cuentas Satélite del Turismo de España, un volumen de demanda equiparable al turismo internacional.

El resto del artículo se estructura de la siguiente manera. En el apartado 2 se resumen las principales perspectivas de análisis para la evaluación de las consecuencias del cambio climático sobre el turismo. En el apartado 3 se presentan los fundamentos de la metodología elegida, los datos y las principales características de la muestra utilizada para la aplicación empírica. En el apartado 4 se muestran los resultados de la estimación y la evaluación de los efectos de un incremento en la temperatura media. Finalmente, en el apartado 5, se resumen los principales resultados y se presentan las conclusiones del trabajo.

\section{REVISIÓN DE LA LITERATURA}

\subsection{Efectos del cambio climático sobre el turismo}

Los trabajos publicados hasta hoy con el fin de analizar los efectos del cambio climático sobre el turismo permiten identificar dos principales perspectivas o líneas de investigación. La primera de ellas se escapa, probablemente, de lo que puede entenderse como análisis económico y se centra fundamentalmente en medir el atractivo turístico desde un punto de vista físico. Un ejemplo claro dentro de esta primera línea se encuentra representado por aquellos trabajos 
ligados al turismo de deportes de invierno y centrados en medir las consecuencias sobre las condiciones de nieve esperadas (Scott et al., 2003 y 2007). Pero también las condiciones idóneas para el turismo en general (Amelung et al., 2007) y para le turismo de playa en particular (Moreno y Amelung, 2009) han tenido su evaluación dentro de esta perspectiva, siendo posible la creación de indicadores sobre pérdida o ganancia de atractivo turístico en términos de las condiciones climáticas esperadas.

La segunda línea de investigación está caracterizada por la implementación de diferentes herramientas utilizadas en el contexto del análisis económico. Dentro de esta segunda línea se distinguen, a su vez, diferentes grupos de trabajo. Así, un primer grupo incluye aquellos trabajos que, siguiendo el camino marcado por los estudios que persiguen evaluar las repercusiones del cambio climático a través de modelos de equilibrio general, definen al turismo como un sector económico más dentro de las cuentas económicas nacionales, tratando así de evaluar las consecuencias monetarias del calentamiento global sobre la industria (Berrittella et al. 2006). En este contexto cabe destacar que la obtención de resultados fiables se ve limitada, no sólo por las dificultades de definir y aplicar las incertidumbres propias de los escenarios de cambio climático a un modelo de equilibrio general, sino también por las dificultades ligadas a definir correctamente el sector turístico en el marco de las cuentas económicas nacionales donde no aparece directamente el sector.

El segundo grupo de estudios, enmarcado en el contexto del análisis de las series temporales, trata de evaluar la sensibilidad de determinadas variaciones de la demanda turística a condiciones meteorológicas especiales. Analíticamente, estos modelos de series temporales no difieren de los aplicados tradicionalmente más allá del uso de datos de mayor frecuencia y de la incorporación de las variables meteorológicas como elemento explicativo de la demanda. Ejemplos de la aplicación de estos modelos de series temporales pueden encontrarse en Agnew y Palutikof (2006), Meyer y Dewar (1999), Moen y Fredman (2007), Rosselló et al. (2010), Rosselló (2011) y Subak et al. (2000).

Finalmente, es posible identificar un tercer grupo de estudios donde el objeto de análisis no es tanto la cuantificación del impacto en términos de número de turistas (o gasto turístico) sino evaluar los posibles efectos del cambio climático sobre la distribución geográfica de los flujos turísticos. En este sentido, cabe citar el estudio pionero de Maddison (2001) donde se analizan las salidas de turistas británicos con un modelo explicativo donde aparece la temperatura como determinante significativo y donde, en consecuencia, es posible simular los posibles efectos del calentamiento global sobre la distribución geográfica de los destinos elegidos por los turistas británicos. En esta misma línea, Lise y Tol (2002) analizan las salidas de turistas holandeses estimando incluso una temperatura óptima para la realización de actividades turísticas. Con una pers- 
pectiva global Hamilton et al. (2005a y 2005b), Bigano et al. (2006) y Hamilton y Tol (2007) utilizan el Hamburg Tourism Model para determinar los factores que determinan los flujos internacionales de turistas a nivel mundial tomando como referencia tanto las salidas como las llegadas e incluyendo las temperaturas como factor explicativo. Los resultados permiten evaluar las consecuencias del crecimiento poblacional, económico y de temperaturas previstas en diferentes escenarios sobre la distribución de los turistas internacionales a nivel mundial.

Dentro de este tercer grupo de estudios, dedicados al análisis de la distribución de los flujos turísticos, han aparecido recientemente diversos trabajos que han adoptado un punto de vista individual (Eugenio-Martín y Campos-Soria, 2010; Bujosa y Rosselló, 2011). Estos trabajos, que constituyen una alternativa a la perspectiva agregada habitualmente utilizada en la literatura, hacen uso de los modelos de elección discreta para determinar la función indirecta de utilidad de los individuos a partir de variables como, por ejemplo, las condiciones climáticas, y evaluar así como cambios en dichas variables pueden repercutir en la utilidad de los individuos y provocar cambios en la elección del destino turístico.

Este estudio, que se enmarca dentro de este último subgrupo de trabajos, considera los viajes interiores de los españoles hacia destinos costeros diferenciando, asimismo, los viajes en función de la temporada en la que se han realizado (temporada alta, media y baja). De esta manera, se toma como referencia el turismo doméstico, un sector frecuentemente marginado en los estudios de esta índole a pesar de representar un peso relativo similar al del turismo internacional (Bigano et al., 2004), a la vez que se desglosa el componente estacional de los viajes turísticos. Al mismo tiempo, se refuerza la idoneidad de aplicar los modelos de elección discreta en el contexto del cambio climático.

\subsection{Modelización de la demanda turística en el contexto de la elección de destino}

La modelización de la demanda sigue siendo uno de los temas de estudio más recurrentes dentro de la literatura turística. Las diferentes revisiones aparecidas recientemente (Lim, 1999; Li et al., 2005; Song and Li, 2008) muestran como en la estimación y predicción de la demanda turística los factores económicos (renta y precios, principalmente) siguen jugando un papel fundamental a la hora de plantear y estimar los modelos. En este sentido, en la mayoría de ocasiones, aunque el fundamento teórico de los modelos planteados haga referencia a como la utilidad de los individuos depende, precisamente, de la renta, los precios y otro tipo de variables económicas, la práctica habitual 
suele desembocar en la búsqueda de correlaciones entre diferentes variables en el contexto del análisis de series temporales.

La consideración de la teoría de la utilidad dentro del contexto de las decisiones turísticas, formalmente descrita por primera vez en Morley (1992), introduce un nuevo marco de análisis permitiendo la consideración de diferentes perspectivas de la decisión del viaje y la introducción de un número mayor de determinantes. De esta manera, individuos o familias con exactamente los mismos perfiles sociodemográficos pueden elegir diferentes destinos, diferentes tiempos de estancia o diferentes niveles de gasto turístico. Sin embargo, más allá de la consideración de la teoría de la utilidad a través de los modelos de elección discreta, se reconoce la existencia de diferencias en las preferencias entre los turistas y que la decisión final no suele ser una elección independiente, aunque sí la última de las decisiones tomadas por el individuo. En este sentido Eugenio-Martin (2003) sugiere que una vez el turista ha decidido irse de viaje, el presupuesto y el medio de transporte elegirá el destino condicionado por sus características individuales y por los atributos de las alternativas que tenga disponibles en el conjunto de elección.

Esta nueva perspectiva microeconómica de modelización de la demanda turística resulta de gran interés para diferentes agentes debido a la alta potencialidad para identificar los determinantes de la elección del destino por parte de los individuos. Este análisis puede dar a conocer, por ejemplo, que los residentes de una región particular están menos interesados en viajar que residentes de otras regiones debido a diferentes aspectos tales como diferencias en el nivel de ingresos, diferencias en instalaciones especificas, o diferencias en atributos ambientales consideradas. En cualquier caso, es importante remarcar como la elección del destino se inscribe como un etapa más en el complejo árbol de decisión llevada a cabo por el turista, siendo el número de variables que pueden llegar a intervenir en esa decisión muy amplio (Correia et al., 2007; Huybers, 2005; Nicolau and Más, 2005; Pestana et al., 2008; Thrane, 2008)

\section{METODOLOGÍA Y DATOS}

\subsection{El modelo de elección discreta para múltiples destinos}

Los modelos de elección discreta, basados en la teoría neoclásica de las características de Lancaster (1966), asumen que la utilidad proporcionada por un determinado bien a los consumidores del mismo puede ser descompuesta en función de las características o atributos que definen ese bien. De esta manera, los modelos de elección discreta ofrecen un marco idóneo para modelizar las elecciones tomadas por los individuos cuando éstos se ven obligados a elegir una opción de entre un conjunto de alternativas mutuamente excluyentes. 
Sin embargo, al ser la utilidad un elemento no observable por el investigador, ésta debe ser modelizada considerando la existencia de un componente aleatorio de la misma (Ben-Akiva and Lerman, 1985). En este contexto, desde el desarrollo del primer modelo de utilidad aleatoria (RUM, en sus siglas en inglés) introducido por McFadden (1974) y conocido como logit condicional, la literatura especializada en la elección discreta ha desarrollado diversas alternativas entre las que cabe destacar el modelo anidado (nested logit) o el modelo de parámetro aleatorio (random parameter logit). En cualquier caso, y a pesar de las ventajas de este último modelo -entre las que se encuentran la posibilidad de considerar las preferencias heterogéneas de los individuos así como la posible correlación entre aquellas alternativas no independientes (McFadden y Train, 2000)-, los datos utilizados en este estudio no han permitido hacer uso de este modelo (para más detalles véase el apartado 4).

En el modelo logit condicional, la utilidad $U_{n i}$ que un individuo (turista en nuestro caso) $n$ recibe de la elección de visitar un destino $i$ en una determinada ocasión de elección, dado un conjunto de elección de alternativas $i=1, \ldots, I$, puede representarse mediante la función indirecta de utilidad que, siguiendo una especificación lineal, toma la siguiente forma:

$$
U_{n i}=\beta^{\prime} x_{n i}+\varepsilon_{n i}
$$

donde $\beta^{\prime} x_{n i}$ es la parte no estocástica de la utilidad indirecta recibida durante la ocasión de elección si el destino $i$ es visitado. De esta manera, $x_{n i}$ son los atributos observados que caracterizan las alternativas disponibles para el turista y $\beta^{\prime}$ es el vector de coeficientes estimados para cada uno de los atributos que entran en $x_{n i}$. Finalmente, el término de error $\varepsilon_{n i}$ captura la variación en las preferencias de los distintos turistas. Así, asumiendo que el turista visita el destino que le reporta la mayor utilidad posible, la probabilidad $\pi_{n i}$ de elegir la alternativa $i$ viene dada por la siguiente expresión:

$$
\pi_{n i}=\operatorname{Pr}\left(\beta^{\prime} x_{n i}+\varepsilon_{n i}>\beta^{\prime} x_{n j}+\varepsilon_{n j}\right) \quad \forall j \neq i
$$

En este punto, y siguiendo los supuestos comúnmente adoptados en la literatura especializada (McFadden, 1978; Train, 2003), se asume que el término de error $\varepsilon_{n i}$ está independiente e idénticamente distribuido según una distribución acumulada de valor extremo tipo I o Gumbel $F\left(\varepsilon_{n i}\right)=\exp \left(-\exp \left(-\varepsilon_{n i}\right)\right)$, por lo que la probabilidad de elegir un determinado destino i en la ecuación (2) puede expresarse como: 


$$
\pi_{n i}=\frac{e^{\beta^{\prime} x_{n i}}}{\sum_{j=1}^{I} e^{\beta^{\prime} x_{n j}}}
$$

Finalmente, el logaritmo de la función de verosimilitud para un valor dado del vector de parámetros $\beta$ toma la forma:

$$
L L(\beta)=\sum_{n=1}^{N} \sum_{i=1}^{I} y_{n i} \log \pi_{n i}(\beta)
$$

Donde $N$ representa el número de turistas en la muestra, $\pi_{n i}(\beta)$ son las probabilidades de elección definidas en la ecuación (3) e $y_{n i}$ es una variable que toma valor uno cuando el turista $n$ elige el destino $i$ y cero en el resto de casos.

Una vez estimado el modelo, el vector de parámetros $\beta$ puede ser utilizado para simular los cambios en las probabilidades de elección de los destinos ante cambios en alguno de los atributos que determinan la utilidad de los turistas. De esta manera, este tipo de análisis de elección resulta útil, no sólo para explicar la elección de destino turístico realizada por los turistas, sino también para evaluar los efectos futuros de hipotéticos cambios en las variables explicativas como, por ejemplo, la temperatura media en las provincias de destino.

\subsection{Datos}

La estadística de Movimientos Turísticos de los Españoles (Familitur), elaborada desde el año 1996 por el Instituto de Estudios Turísticos, constituye una fuente de datos de gran valor para cuantificar y caracterizar los viajes que los residentes españoles realizan a las distintas Comunidades Autónomas del país y también al extranjero. El universo de estudio de Familitur incluye a todos los individuos que residen en su primera vivienda a lo largo de todo el territorio nacional a excepción de Ceuta y Melilla. Más concretamente, Familitur recoge información referente a las características básicas de los viajes -tales como el destino, el tipo de alojamiento, el medio de transporte, etc.- de un panel de hogares formado por unos 40.000 individuos agrupados en 12.400 familias. La selección de estos hogares se realiza mediante un proceso de muestreo bietápico, con una selección proporcional de las secciones censales del Instituto Nacional de Estadística (unidades de la primera etapa de muestreo) y de las primeras residencias que conforman la segunda etapa de muestreo.

Aunque el nivel de desagregación de la información contenida en Familitur aparece en términos de viaje-individuo (es decir, aparece información para cada individuo que realiza un viaje, aunque este sea compartido por varios miembros 
de la misma familia), es frecuente enmarcar las decisiones de naturaleza turística utilizando el hogar (o unidad familiar) como unidad de análisis (EugenioMartín, 2003). De esta manera, la información contenida en las más de 120.000 observaciones de la base de datos de Familitur (en forma de viajes-individuo) ha permitido obtener un total de 64.768 viajes-familia, cifra que representa los viajes realizados durante el año 2005 por los hogares que conforman la muestra de esta base de datos. ${ }^{1}$

Dado que el propósito de este trabajo es analizar el papel de las temperaturas como elemento explicativo de la distribución de los viajes realizados por los españoles a provincias costeras peninsulares, es preciso seleccionar tan sólo aquellas observaciones que se enmarquen dentro de este objetivo. Por este motivo, con el fin de considerar sólo las alternativas reales de elección de las familias, únicamente se han incluido aquellas observaciones correspondientes a los viajes entre provincias (excluyendo los desplazamientos dentro de las misma provincia), realizados a provincias costeras de la península (excluyendo los viajes desde y hacía las Islas Baleares y las Islas Canarias). Por otra parte, y con el fin de descartar aquellos viajes en los que el clima puede no jugar un papel determinante, se han incluido sólamente los viajes cuyo motivo principal estuviera relacionado con el ocio, las vacaciones o la práctica de deportes. El resultado final implica la consideración de los viajes interiores realizados por los españoles a alguna de las 19 provincias costeras peninsulares, lo que supone una muestra de 9.218 viajes, de los cuales el $54,50 \%$ corresponde a desplazamientos realizados durante la temporada alta, el $29,69 \%$ durante la temporada media y el $15,81 \%$ restante a la temporada baja.

Asimismo la identificación de los determinantes de la elección de destino turístico ha requerido de un minucioso proceso de recogida de información para poder caracterizar cada una de las provincias costeras consideradas en la aplicación. Por este motivo, la base de datos de este estudio se ha completado a partir de la recopilación de información sobre la disponibilidad y calidad de playas y otros espacios naturales en cada una de las provincias, la dotación de servicios públicos para fines turísticos al entorno de las playas (banderas azules, posibilidad de anclaje, playa urbana, tipo de arena, etc.), la dotación de recursos culturales con posible uso turístico (museos, etc.), las condiciones meteorológicas promedio en cada una de las provincias y meses del estudio (temperatura, precipitación y humedad), etc. Es importante destacar que esta información, recogida de diversas fuentes como el Ministerio de Medio Ambiente, Medio Rural y Marino o la Agencia Estatal de Meteorología (AEMET), ha sido agre-

\footnotetext{
1 Para obtener información sobre los viajes-individuo y viajes-familia efectuados por los españoles durante el año 2005 o en años posteriores pueden consultarse los informes anuales que periódicamente publica el Instituto de Estudios Turísticos en su página web (http://www.iet.tourspain.es/).
} 
gada a nivel provincial para recoger los valores promedios de cada variable en cada una de las provincias de estudio. Finalmente, la utilización de un sistema de información geográfica ha permitido recoger información sobre la distancia existente entre las capitales de provincia incluidas en el estudio que se ha incorporado como una variable explicativa más del proceso de elección de destino turístico.

\section{RESULTADOS Y SIMULACIÓN}

\subsection{Resultados de la estimación}

A pesar del elevado número de variables consideradas inicialmente como posibles determinantes de la elección del destino turístico, en la Tabla 1 aparecen las que finalmente se han incluido en el modelo debido a su significación estadística. Cabe destacar que muchos de los servicios turísticos ofrecidos en las playas están áltamente correlacionados entre ellos y con algunas variables ambientales que miden el atractivo turístico de la zona, por lo que ha sido necesario tener en cuenta el patrón de correlaciones existente entre las variables explicativas a la hora de especificar el modelo de elección discreta.

Tabla 1

Descripción de las variables consideradas

\begin{tabular}{llcccc}
\hline \multicolumn{1}{c}{ Variable } & \multicolumn{1}{c}{ Descripción } & Mínimo & Media & Máximo & $\begin{array}{c}\text { Desviación } \\
\text { estándar }\end{array}$ \\
\hline Longitud & $\begin{array}{l}\text { Longitud total de las playas } \\
\text { de la provincia (en metros) }\end{array}$ & 10,59 & 79,83 & 149,35 & 40,31 \\
Playas urbanas & $\begin{array}{l}\text { Porcentaje de playas } \\
\text { cercanas a zonas urbanas }\end{array}$ & 0,00 & 0,20 & 0,74 & 0,20 \\
Bandera azul & $\begin{array}{l}\text { Porcentaje de playas con el } \\
\text { distintivo de bandera azul }\end{array}$ & 0,04 & 0,33 & 1,00 & 0,34 \\
Fondeo & $\begin{array}{l}\text { Porcentaje de playas con } \\
\text { posibilidad de fondeo }\end{array}$ & 0,06 & 0,36 & 0,78 & 0,17 \\
Distancia & $\begin{array}{l}\text { Distancia entre la provincia } \\
\text { de origen y la de destino (en } \\
\text { kilómetros) }\end{array}$ & 65,00 & 631,90 & 1385,00 & 285,17 \\
Temperatura T-Alta & $\begin{array}{l}\text { Temperatura media } \\
\text { temporada alta }\end{array}$ & 16,75 & 21,62 & 24,90 & 2,69 \\
Temperatura T-Media & $\begin{array}{l}\text { Temperatura media } \\
\text { temporada media }\end{array}$ & 10,68 & 14,78 & 17,55 & 1,92 \\
Temperatura T-Baja & $\begin{array}{l}\text { Temperatura media } \\
\text { temporada baja }\end{array}$ & 7,10 & 10,94 & 13,90 & 1,80 \\
\hline
\end{tabular}

(*) Temperaturas calculadas a partir de los datos del periodo 1971-2000 (en ${ }^{\circ} \mathrm{C}$ ).

Fuente: Elaboración propia a partir de los datos del Ministerio de Medio Ambiente, Medio Rural y Marino y AEMET. 
Los resultados econométricos del modelo logit condicional descrito en el apartado 2.2 estimado mediante el paquete estadístico MatLab a través de una estimación por máxima verosimilitud se presentan en la Tabla $2 .^{2}$ Los resultados permiten comprobar como la bondad de ajuste del modelo $\left(\mathrm{R}^{2}\right.$ de McFadden del 28,03\%) se sitúa en los niveles habituales para este tipo de modelos de elección discreta según los contrastes basados en el ratio de verosimilitud. $^{3}$

Asimismo, los signos y coeficientes obtenidos en la estimación del modelo corresponden con las expectativas previas existentes para cada una de las variables. De esta manera, el signo negativo de los coeficientes de las variables 'distancia' y porcentaje de 'playas urbanas' muestran el efecto de estos atributos sobre la probabilidad de elección de un destino en particular, en el sentido de que a mayor distancia (o mayor porcentaje de playas urbanas) menor probabilidad de eleción. Por el contrario, el signo positivo del coeficiente de variables como la 'longitud' de las playas, el porcentaje de playas con distintivo de 'bandera azul' o con posibilidades de 'fondeo' pone de manifiesto el efecto positivo de estos atributos sobre la probabilidad de elección de destino turístico.

Con el fin de poder analizar el efecto que ejercen las temperaturas en el proceso de elección de destino turístico y observar, asimismo, como este efecto varía a lo largo de las tres temporadas turísticas (alta, media y baja), ${ }^{4}$ se han incluido en el modelo tres variables para recoger la temperatura promedio en cada uno de estos tres periodos. Así, para cada observación incluida en la muestra del estudio, estas variables toman el valor de la temperatura promedio de la temporada si la observación corresponde a un viaje realizado en esa temporada, y el valor cero en el resto de casos. Para tratar de capturar las relaciones no lineales que puedan existir entre la temperatura y la probabilidad de elección de un destino, de manera similar a otros estudios (Maddison, 2001; Hamilton et al., 2005a), se han incluido los términos cuadráticos de estas variables de temperatura. De esta manera, la significación estadística de los coeficientes estimados en el modelo logit condicional para los términos cuadráticos ponen de manifiesto, en este caso también, la presencia de esa relación no lineal.

\footnotetext{
${ }^{2}$ Cabe destacar que durante la estimación del modelo aquí presentado se ha tenido en cuenta también la posible existencia de preferencias heterogéneas entre los turistas hacia distintos determinantes de la elección de destino turístico. En cualquier caso, la estimación de diversos modelos de parámetro aleatorio ha permitido rechazar, para este estudio concreto, la hipótesis de heterogeneidad de preferencias entre los turistas.

${ }^{3}$ Estos contrastes, conocidos con el nombre de $\mathrm{R}^{2}$ de McFadden (o Pseudo- $\mathrm{R}^{2}$ ) y $\mathrm{R}^{2}$ de McFadden ajustado, fueron introducidos por McFadden (1974) y por Ben-Akiva y Lerman (1985), respectivamente.

${ }^{4}$ La temporada alta corresponde a los meses de junio, julio, agosto y septiembre; la temporada media con los meses de marzo, abril, mayo y octubre; y la temporada baja con los meses de enero, febrero, noviembre y diciembre.
} 
Tabla 2

Resultados del modelo de elección discreta

\begin{tabular}{lcc}
\hline \multicolumn{1}{c}{ Variable } & Coeficiente & Error estándar \\
\hline Longitud & 0,0146 & 0,0004 \\
Playas urbanas & $-0,4633$ & 0,1133 \\
Bandera azul & 0,5172 & 0,0406 \\
Fondeo & 0,5657 & 0,0576 \\
Distancia & $-0,5640$ & 0,0064 \\
Temperatura T-Alta & 1,3394 & 0,1664 \\
Temperatura T-Media & 0,6648 & 0,2203 \\
Temperatura T-Baja & $-0,5286$ & 0,1805 \\
Temperatura T-Alta cuadrado & $-0,0313$ & 0,0039 \\
Temperatura T-Media cuadrado & $-0,0250$ & 0,0075 \\
Temperatura T-Baja cuadrado & $0,0176\left(^{*}\right)$ & 0,0087 \\
$\mathrm{R}^{2}$ de McFadden & 0,2803 & \\
$\mathrm{R}^{2}$ de McFadden Ajustado & 0,2799 & \\
\hline
\end{tabular}

Todos los coeficientes son significativos al $1 \%$ con la excepción de aquellos indicados con ${ }^{(*)}$ que sólo son significativos al $5 \%$.

Fuente: Elaboración propia.

Figura 1

Utilidad marginal de la temperatura

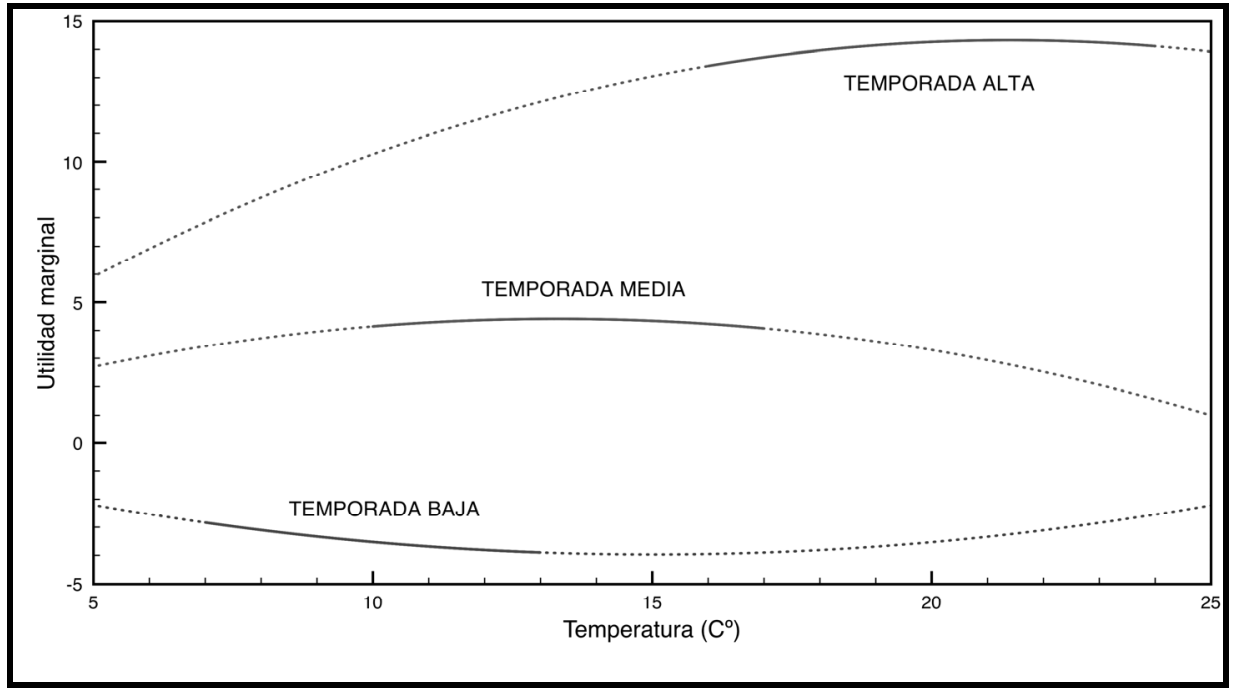

Fuente: Elaboración propia. 
Los resultados permiten constatar como un aumento de la temperatura media durante la temporada alta y media, más allá de los valores actualmente registrados y representados mediante las líneas continuas, provocaría una reducción de la utilidad marginal y, por ende, de la probabilidad de elección de destino turístico en aquellas provincias que ya registran en la actualidad temperaturas por encima del promedio del resto de provincias costeras, como por ejemplo las provincias costeras del Mediterráneo. Sin embargo, se observa que, para aquellas provincias con temperaturas más bajas durante las temporadas alta y media, existe un potencial de atracción dada su relación positiva con la utilidad marginal que caracteriza el primer tramo de las curvas. En cualquier caso, es importante señalar como, para el caso de la temporada media, las variaciones de la utilidad marginal provocadas por cambios de temperatura son menores que en la temporada alta.

Para el caso de la temporada baja, se obtiene que el incremento de la temperatura supone un descenso de la utilidad marginal del turista, dando a entender que, durante el invierno, gozar de temperaturas menores supone un incremento de la probabilidad de visita para las provincias de costa españolas, con lo que un incremento de las temperaturas supondría una perdida de atractivo turístico.

\subsection{Simulación}

Al objeto de evaluar las consecuencias sobre la distribución de los flujos turísticos interiores en España de un incremento en las temperaturas, en este apartado se utilizan los resultados de la estimación para simular dos incrementos de temperatura promedio. El primero de ellos hace referencia a un incremento en la temperatura de $1^{\circ} \mathrm{C}$ en cada un de las temporadas. La segunda hace referencia al incremento en la temperatura también de $1^{\circ} \mathrm{C}$ pero acontecido únicamente en la temporada alta. Esta discriminación se debe a los informes publicados por el Ministerio de Medio Ambiente ${ }^{5}$ en donde se pone de manifiesto la posibilidad de que se produzca un incremento de temperaturas superior en verano que en invierno, cualquiera que sea el escenario de cambio climático de referencia.

A la hora de interpretar los resultados obtenidos por la simulación es importante señalar que únicamente la variable objeto de estudio, esto es la temperatura, sufre algún cambio y, por lo tanto, el resto de atributos que determinan la elección de destino turístico permanecen inalterados. Consecuentemente, el ejercicio de simulación aquí propuesto no considera posibles cambios

\footnotetext{
${ }^{5}$ Véase, por ejemplo: Ministerio de Medio Ambiente. Informe para el Presidente del Gobierno elaborado por expertos en cambio climático [en línea].

$<$ http://www.marm.es/es/cambio-climatico/publicaciones/documentacion/ad_hoc_resumen_tcm712460.pdf $>$ [Consultado: 20 agosto 2011].
} 
simultáneos de dos o más variables que determinan la elección. Por otra parte, la simulación supone un incremento de la temperatura homogéneo en todo el territorio español, por lo que no se consideran diferencias geográficas en el incremento de las temperaturas.

La Tabla 3 presenta los resultados de la simulación en que aparecen los dos escenarios considerados. En líneas generales se puede observar como las provincias costeras más cálidas localizadas en el Mediterráneo (a excepción de Granada, Barcelona y Girona), se verían negativamente afectadas por un aumento de las temperaturas (tanto para un aumento de temperaturas en temporada alta como para un aumento de las temperaturas para todo el año). En este sentido cabe recordar que la mayor parte de los viajes turísticos de los españoles se ralizan durante el verano, por lo que es normal que la tendencia del verano acabe arrastrando el comportamiento agregado del año. En el caso opuesto, las provincias costeras más frías del norte peninsular incluyendo Barcelona y Girona se verían positivamente afectadas, por lo que su capacidad de atracción de turistas aumentaría en ambos escenarios.

Tabla 3

Probabilidad de elección de destino turístico por provincias

\begin{tabular}{|c|c|c|c|c|c|}
\hline Provincia & $\begin{array}{l}\text { Predicción } \\
\text { inicial } \\
(\%)\end{array}$ & $\begin{array}{c}\text { Aumento } 1 \mathrm{C}^{\circ} \\
\text { en } \\
\text { todas las } \\
\text { temporadas } \\
(\%)\end{array}$ & Variación & $\begin{array}{c}\text { Aumento } 1 \mathrm{C}^{\circ} \\
\text { en temporada } \\
\text { alta } \\
(\%)\end{array}$ & Variación \\
\hline Alicante & $9,65 \%$ & $9,18 \%$ & $-0,0048$ & $9,26 \%$ & $-0,0039$ \\
\hline Almería & $2,88 \%$ & $2,69 \%$ & $-0,0018$ & $2,72 \%$ & $-0,0016$ \\
\hline Barcelona & $8,33 \%$ & $8,42 \%$ & 0,0008 & $8,38 \%$ & 0,0005 \\
\hline Cádiz & $5,84 \%$ & $5,66 \%$ & $-0,0018$ & $5,69 \%$ & $-0,0015$ \\
\hline Castellón & $6,31 \%$ & $6,03 \%$ & $-0,0027$ & $6,08 \%$ & $-0,0023$ \\
\hline A Coruña & $5,69 \%$ & $6,13 \%$ & 0,0044 & $6,07 \%$ & 0,0038 \\
\hline Girona & $6,02 \%$ & $6,20 \%$ & 0,0017 & $6,16 \%$ & 0,0014 \\
\hline Granada & $2,48 \%$ & $2,50 \%$ & 0,0002 & $2,49 \%$ & 0,0002 \\
\hline Guipúzcoa & $3,47 \%$ & $3,61 \%$ & 0,0013 & $3,60 \%$ & 0,0012 \\
\hline Huelva & $2,91 \%$ & $2,80 \%$ & $-0,0011$ & $2,83 \%$ & $-0,0009$ \\
\hline Lugo & $1,94 \%$ & $2,20 \%$ & 0,0025 & $2,17 \%$ & 0,0023 \\
\hline Málaga & $4,99 \%$ & $4,87 \%$ & $-0,0012$ & $4,88 \%$ & $-0,0010$ \\
\hline Murcia & $5,58 \%$ & $5,11 \%$ & $-0,0047$ & $5,19 \%$ & $-0,0039$ \\
\hline Asturias & $4,21 \%$ & $4,73 \%$ & 0,0052 & $4,62 \%$ & 0,0041 \\
\hline Pontevedra & $2,61 \%$ & $2,67 \%$ & 0,0006 & $2,67 \%$ & 0,0006 \\
\hline Cantabria & $5,27 \%$ & $5,68 \%$ & 0,0041 & $5,61 \%$ & 0,0034 \\
\hline Tarragona & $10,00 \%$ & $9,89 \%$ & $-0,0011$ & $9,89 \%$ & $-0,0011$ \\
\hline Valencia & $7,48 \%$ & $7,07 \%$ & $-0,0041$ & $7,15 \%$ & $-0,0033$ \\
\hline Vizcaya & $4,33 \%$ & $4,57 \%$ & 0,0023 & $4,53 \%$ & 0,0020 \\
\hline
\end{tabular}

Fuente: Elaboración propia. 
Finalmente, cabe destacar el efecto negativo observado en Murcia y en las provincias valencianas y andaluzas, con la excepción de Granada. Esta última provincia podría romper la tendencia general observada por su especial atractivo en términos de turismo de montaña.

\section{CONCLUSIONES}

A pesar de la incertidumbre sobre los cambios sociales que acompañarán el calentamiento global durante las próximas décadas existe un creciente interés por anticipar los efectos que el cambio climático puede acarrear sobre los principales sectores económicos. El turismo, como actividad económica altamente sensible a las condiciones climáticas, está empezando a suscitar el interés de la literatura de referencia. Las diferentes perspectivas utilizadas para evaluar las consecuencias del cambio climático coinciden en señalar una pérdida de mercado para los destinos templados que desincentivaría los tradicionales flujos de norte a sur que vienen reproduciéndose cada año desde el surgimiento del turismo de masas.

Aunque los resultados aparecidos hasta el momento sitúan a España en un escenario claramente negativo, cabe apuntar que los modelos desarrollados hasta el momento se han basado principalmente en datos agregados internacionales y anuales, lo que dificulta el análisis en profundidad de un sector caracterizado por una marcada estacionalidad y distribuido heterogéneamente por el territorio español que, además, presenta diferencias significativas en términos de condiciones climáticas.

Tomando como referencia el mercado interior, los resultados empíricos del modelo de elección discreta estimado en este artículo han permitido identificar la temperatura como un factor relevante para determinar la probabilidad de visitar un determinado destino de costa peninsular específico. Asimismo, la existencia de efectos no lineales entre la temperatura y las probabilidades de elección ha permitido identificar, para cada una de las temporadas definidas, los umbrales de temperatura más allá de los cuales un aumento de las temperaturas puede derivar en un incremento o descenso (según el caso) de la utilidad marginal de la visita y, por consiguiente, de la probabilidad de elección del destino.

Los resultados generales muestran como en temporada alta, las provincias costeras situadas en el rango alto de temperaturas podrían ver perjudicada su cuota de mercado a favor de aquellas provincias con temperaturas menores, ante un escenario de aumento generalizado de la temperatura. Durante la temporada media, aunque los resultados son similares, se aprecia un menor efecto sobre la utilidad, dando a entender que el potencial de pérdida/incremento de atractivo climático es menor. Finalmente, en la temporada baja, se observa como un incremento en la temperatura afectaría negativamente a la utilidad de los turistas en todas las provincias. El tramo de utilidad decreciente en el que se encuentran 
las provincias españolas podría relacionarse con la existencia de numerosas estaciones de esquí, situadas precisamente en provincias españolas costeras, que verían mermado su atractivo ante un escenario de aumento de las temperaturas.

En cualquier caso, el balance general anual efectuado a través de las dos simulaciones apunta como las provincias costeras más cálidas se verían perjudicadas por un aumento de las temperaturas mientras que las más frías podrían verse beneficiadas de un aumento de las temperaturas.

Las investigaciones futuras deberán centrarse en analizar el papel del cambio climático en los flujos de turismo tomando como referencia diferentes segmentos de mercado y no sólo el dirigido a las zonas costeras. Al mismo tiempo, las diferencias entre las regiones deberán ser investigadas en el contexto de diferentes escenarios de cambio climático que consideren, no sólo los cambios esperados en las variables climáticas, sino también las especificidades de cada región.

\section{REFERENCIAS BIBLIOGRÁFICAS}

AGNEW, M., y PALUTIKOF, J. (2006): "Impacts of short-term climate variability in the UK on demand for domestic and international tourism" en Climate Research, 21: pp 109-120.

AMELUNG, B.; NICHOLLS, S. y VINER, D. (2007): "Implications of global climate change for tourism flows and seasonality" en Journal of Travel Research, 45: pp 285-296.

BEN-AKIVA, M., y LERMAN, S.R. (1985): Discrete choice analysis: theory and application to travel demand, pp. 390, Cambridge, Massachusetts: The MIT Press.

BERRITTELLA, M.; BIGANO, A.; ROSON, R. y TOL, R. (2006): "A general equilibrium analysis of climate change impacts on tourism" en Tourism Management, 27: pp 913-924.

BIGANO, A., HAMILTON, J., y TOL, R. (2006): "The impact of climate holiday destination choice" en Climatic Change, 76: pp 389-406.

BIGANO, A.; HAMILTON, J.; LAU, M.; TOL, R.S.J., y ZHOU, Y. (2004): A global database of domestic and international tourist numbers at national and subnational level, Research unit Sustainability and Global Change FNU-54, Hamburg University and Centre for Marine and Atmospheric Science, Hamburg.

BUJOSA, A. y ROSSELLÓ, J. (2011): Climate change and summer mass tourism: The case of Spanish domestic tourism. (mimeo) 
CORREIA, A.; SANTOS, C.M.; y BARROS, C.P. (2007): "Tourism in Latin America. A choice analysis" en Annals of Tourism Research, 34: pp 610629.

EUGENIO-MARTÍN, J.L. (2003): "Modelling determinants of tourism demand as a five-stage process: A discrete choice methodological approach" en Tourism and Hospitality Research 4: pp 341-354.

EUGENIO-MARTIN, J.L. y CAMPOS-SORIA, J.A. (2010): "Climate in the region of origin and destination choice in outbound tourism demand" en Tourism Management 31: pp 744-753.

HAMILTON, J., MADDISON, D. y TOL, R. (2005a): "Climate change and international tourism: A simulation study" en Global Environmental Change, 15: pp 253-266.

HAMILTON, J., MADDISON, D. y TOL, R. (2005b): "The effects on climate change on international tourism" en Climate Research, 29: pp 245-254.

HAMILTON, J., y TOL, R. (2007): "The impact of climate change on tourism in Germany, the UK and Ireland: A simulation study" en Regional Environmental Change, 7: pp161-172.

HEIN, L. (2007): The Impact of Climate Change on Tourism in Spain. CICERO Working Paper 2007:02

HUYBERS, T. (2005): "Destination choice modelling: What's in a name?" en Tourism Economics, 11: 329-350.

LANCASTER, K.J. (1966): "A New Approach to Consumer Theory" en The Journal of Political Economy, 74: pp 132-157.

LI, G.; SONG, H. y WITT, S.F. (2005): "Recent developments in econometric modeling and forecasting" en Journal of Travel Research, 44, pp 82-99.

LIM, C. (1999): "A meta analysis review of international tourism demand" en Journal of Travel Research, 37: pp 273-284

LISE, W., y TOL, R. (2002): "Impact of climate on tourism demand" en Climatic Change, 55: pp 429-449.

MADDISON, D. (2001): "In search of warmer climates? The impact of climate change on flows of British tourist" en Climatic Change 49: pp 193-208.

MCFADDEN, D. (1974): "The Measurement of Urban Travel Demand" en Journal of Public Economics, 3: pp 303-328.

MCFADDEN, D. (1978): "Modelling the choice of residential location" en Karlqvist A. (Ed.) Spatial Interaction Theory and Planning Models (pp. 7596). Amsterdam: North-Holland Publishing.

MCFADDEN, D., y TRAIN, K.E. (2000): "Mixed MNL Models for Discrete Response" en Journal of Applied Econometrics, 15: pp 447-470.

MEYER, D. y DEWAR, K. (1999): "A new tool for investigating the effect of weather on visitor numbers" en Tourism Analysis, 4: pp 145-155.

MOEN, J. y FREDMAN, P. (2007): "Effects of cliOmate change in alpine skiing in Sweden" en Journal of Sustainable Tourism, 14: pp 418-437. 
MORENO, A. y AMELUNG, B. (2009): "Climate Change and Tourist Comfort on Europe's Beaches in Summer: A Reassessment" en Coastal Management, 37: pp 550-568.

MORLEY, C.L. (1992): "A microeconomic theory of international tourism demand" en Annals of Tourism Research, 19: pp 250-267.

NICOLAU, J.L., y MÁS, F.J. (2005): "Stochastic modeling. A three-stage tourist choice process" en Annals of Tourism Research, 32: 49-69.

PESTANA, C.; BUTLER, R. y CORREIA, A. (2008): "Heterogeneity in Destination Choice. Tourism in Africa" en Journal of Travel Research, 47: pp 235246

ROSSELLÓ, J (2011): "The impact of the NAO index on European Airline Transit" en Transportation Research Part D: Transport and Environment, 16: 183-187

ROSSELLÓ, J.; RIERA, A. y CÁRDENAS, V. (2010): "The impact of weather variability on British outbound flows" en Climatic Change 105: 281-292.

SCOTT, D.; McBOYLE, G. y MILLS, B. (2003): "Climate change and the skiing industry in southern Ontario (Canada): exploring the importance of snowmaking as a technical adaptation" en Climate Research, 23: pp 171-181.

SCOTT, D.; McBOYLE, G. y MINOGUE, A. (2007): "Climate change and Quebec's ski industry" en Global Environmental Change, 17: pp 181-190.

SONG, H. y LI, G. (2008): "Tourism demand modeling and forecasting: A review of recent research" en Tourism Management 29: pp 203-220.

SUBAK, S.; PALUTIKOF, J.; AGNEW, M.; WATSON, S.; BENTHAM, C.; CANNEL, M.; HULME, M.; MCNALLY, S.; THORNES, J.; WAUGHRAY, D. y WOODS, J. (2000): "The impact of the anomalous weather of 1995 on the UK Economy" en Climatic Change, 44: pp 1-26.

THRANE, C. (2008): "The Determinants of Students' Destination Choice for their Summer Vacation Trip" en Scandinavian Journal of Hospitality and Tourism, 8: pp 333-348.

TRAIN, K.E. (2003): Discrete choice methods with simulation, pp 336, Cambridge: Cambridge University Press. 